\title{
Efeitos do exercício físico sobre o risco cardiovascular e qualidade de vida em idosos hipertensos
}

\author{
Effects of physical exercise on cardiovascular risk and quality of life \\ in hypertensive elderly people
}

\author{
Andressa do Nascimento Cassiano (https://orcid.org/0000-0002-1463-0244) ${ }^{1}$ \\ Thiago Santos da Silva (https://orcid.org/0000-0002-5616-0658) ${ }^{1}$ \\ Carlos Queiroz do Nascimento (https://orcid.org/0000-0002-3345-2500) ${ }^{2}$ \\ Emília Maria Wanderley (https://orcid.org/0000-0002-9817-7052) ${ }^{3}$ \\ Eduardo Seixas Prado (https://orcid.org/0000-0002-9631-3292) ${ }^{1}$ \\ Táscya Morganna de Morais Santos (https://orcid.org/0000-0002-4798-4890) ${ }^{4}$ \\ Carolina Santos Mello (https://orcid.org/0000-0001-6461-1749) ${ }^{5}$ \\ João Araújo Barros-Neto (https://orcid.org/0000-0002-7603-1095) ${ }^{6}$
}

${ }^{1}$ Instituto de Educação

Física e Esporte,

Universidade Federal

de Alagoas (UFAL). Av.

Lourival Melo Mota $\mathrm{s} / \mathrm{n}$,

Tabuleiro do Martins.

57072-970 Maceió

AL Brasil.

joao.neto@fanut.ufal.br

${ }^{2}$ Escola de Enfermagem e

Farmácia, UFAL. Maceió

AL Brasil.

${ }^{3}$ Pós-Graduação em

Gerontologia, Universidade

Estadual de Campinas.

Campinas SP Brasil.

${ }^{4}$ Instituto Federal de

Alagoas, Campus Satuba.

Satuba AL Brasil.

${ }^{5}$ Escola de Nutrição,

Universidade Federal da

Bahia. Salvador BA Brasil.

${ }^{6}$ Faculdade de Nutrição,

UFAL. Maceió AL Brasil.

\begin{abstract}
The scope of this article is to evaluate the effects of a mixed protocol of physical exercises on cardiovascular risk (CVR), quality of life and the presence of depressive symptoms in elderly hypertensive patients. It involved a longitudinal, prospective and quasi-experimental study with 48 elderly individuals. A physical exercise protocol was developed for 16 weeks according to American College of Sports Medicine, and anthropometric variables (weight, height, body mass index and waist circumference), as well as biochemical variables (total cholesterol, LDL-c, $H D L-c$; triglycerides) were evaluated. The mean age was $66.5 \pm 4.8$ years. The frequent $C V R$ factors were dyslipidemia (58.3\%) and sedentary lifestyle (45.8\%). After intervention, there was a reduction in mean values of total and LDL-cholesterol and increased HDL-cholesterol levels. The CVR and global risk score decreased significantly. The frequency of depressive symptoms decreased from $25 \%$ to $4.2 \%$. An improvement was observed in SF-36 physical, health and vitality domains. The mixed protocol of physical exercises constituted a possible low cost and effective alternative in the improvement of the lipid profile and the quality of life related to the health of the elderly who participated in this research.
\end{abstract}

Key words Exercise, Cardiovascular Diseases, Quality of life, Depression
Resumo O objetivo deste artigo é avaliar efeitos de um protocolo misto de exercícios físicos sobre o risco cardiovascular (RCV), qualidade de vida e presença de sintomas depressivos em idosos hipertensos. Estudo longitudinal, prospectivo e quase -experimental com 48 idosos. Desenvolveu-se um protocolo de exercícios físicos durante 16 semanas, segundo American College Sports of Medicine e foram realizadas avaliações antropométricas (peso, estatura, indice de massa corporal e circunferência da cintura) e bioquimicas (colesterol total, LDL-c, HDL-c; triglicerídeos). A média de idade foi de 66,5 $\pm 4,8$ anos. Os fatores de RCV frequentes foram dislipidemia $(58,3 \%)$ e sedentarismo (45,8\%). Após intervenção houve redução dos valores médios de colesterol total e LDL-colesterol e aumentaram niveis de HDL-colesterol. O RCV e a pontuação do escore de risco global diminuíram significativamente. A frequência de sintomas depressivos reduziu de $25 \%$ para $4,2 \%$. Observouse melhora nos domínios do SF-36: físico, saúde e vitalidade. O protocolo misto de exercícios físicos constituiu-se em alternativa possivel, de baixo custo e eficaz na melhora do perfil lipidico e da qualidade de vida relacionada à saúde dos idosos desta pesquisa.

Palavras-chave Exercício, Doenças Cardiovasculares, Qualidade de vida, Depressão 


\section{Introdução}

O envelhecimento, antes considerado um fenômeno, hoje faz parte da realidade da maioria das sociedades, sendo esse crescimento mais acentuado nas sociedades ocidentais ${ }^{1}$. Caracteriza-se como um processo multifatorial influenciado por aspectos psicológicos, sociais, biológicos e funcionais, que diminuem a capacidade física e comprometem o desempenho para as atividades de vida diária ${ }^{2}$.

Paralelamente a essa rápida transição demográfica, houve um aumento das doenças crônicas não transmissíveis (DCNT) ${ }^{2}$, de forma que a hipertensão arterial, o colesterol elevado, a obesidade e o diabetes seguem em tendência ascendente, especialmente na população idosa ${ }^{3}$.

$\mathrm{O}$ aparecimento dessas doenças parece estar associado ao comprometimento gradativo da capacidade funcional e à redução da qualidade de vida relacionada à saúde (QVRS), esta última, favorecendo o aparecimento de sintomas depressivos em pessoas idosas ${ }^{4,5}$. A avaliação da QVRS é uma ferramenta de saúde pública importante, principalmente nesta população, tendo em vista o aumento da expectativa de vida com o acúmulo de quadros patológicos, comuns ao processo de envelhecimento ${ }^{1}$.

A hipertensão arterial sistêmica (HA) é a doença crônica de maior incidência no Brasil e no mundo. Considerada o principal fator de risco para doenças cardiovasculares, foi responsável por 33\% dos óbitos no Brasil em 2011 atingindo tanto homens quanto mulheres de diferentes faixas etárias, com maior prevalência nas pessoas idosas $^{6,7}$.

O Ministério da Saúde propõe a estratificação do risco cardiovascular (RCV) para definir o prognóstico e a conduta na abordagem do hipertenso na atenção primária, recomendando o Escore de Risco de Framingham (ERF), o qual, testado com sucesso em várias populações, possibilita estabelecer o risco absoluto de doenças cardiovasculares nos próximos dez anos ${ }^{6}$.

Evidências acumuladas nos últimos anos mostram que as condutas não medicamentosas, como a adoção de programas de exercícios físicos e alimentação adequada, constituem-se em estratégias iniciais na hipertensão leve a moderada. São ações de baixo custo e risco mínimo, capazes de contribuir para a regulação da pressão arterial, tratamento de dislipidemias e da obesidade ${ }^{8,9}$.

O sedentarismo e a HA são importantes e modificáveis fatores de risco para doença cardiovascular e mortalidade ${ }^{10}$. A prática de atividade física contribui com ações de promoção da saúde, que melhoram a QVRS, reduzem a presença de sintomas depressivos e auxiliam no controle da pressão arterial $^{11,12}$.

Indivíduos ativos apresentam risco $30 \%$ menor de desenvolver HA que os sedentários ${ }^{13}$. Entretanto, a redução dos riscos parece estar associada à dedicação, disponibilidade e aderência no programa de exercícios, além do grau da hipertensão e estilo de vida do indivíduo ${ }^{14}$.

A atividade física vem sendo preconizada como uma grande aliada das pessoas idosas para enfrentar o envelhecimento nos contextos físico, psicológico e social ${ }^{15}$. Juntamente com a prática de esportes, constitui-se em tratamento indispensável tanto na prevenção, como na melhora do bem estar geral, da independência e da competência nas atividades diárias ${ }^{3}$.

Desta forma, o presente estudo foi desenvolvido com o objetivo de avaliar os efeitos de um protocolo misto de exercícios físicos sobre o risco cardiovascular, qualidade de vida relacionada à saúde e presença de sintomas depressivos em idosos hipertensos.

\section{Métodos}

Este estudo é parte integrante de um projeto maior denominado "Estado nutricional, capacidade funcional e risco cardiovascular em idosos inseridos em um programa de extensão universitário". Foi desenvolvido por pesquisadores do Laboratório de Nutrição e Metabolismo no Envelhecimento (LANME) da Faculdade de Nutrição da UFAL no período de janeiro a maio de 2015. Trata-se de um estudo longitudinal, prospectivo, do tipo quase experimental, onde os sujeitos envolvidos foram avaliados antes e após a execução de protocolo misto de exercícios físicos, com duração de 16 semanas.

A amostra foi composta por pessoas idosas e hipertensas cadastrados no LANME por meio de um plano amostral não probabilístico de conveniência.

Foram estabelecidos os seguintes critérios de inclusão: idade igual ou superior a 60 anos; diagnóstico clínico de HAS (pressão arterial $\geq 140 / 90$ $\mathrm{mmHg}$ ), seguindo os critérios da I Diretriz Brasileira de Prevenção Cardiovascular ${ }^{16}$; concordância em participar do estudo e assinar o Termo de Consentimento Livre e Esclarecido (TCLE).

Como critérios de exclusão estabeleceram-se: capacidade funcional classificada como desempenho muito ruim, segundo a Short Physical Per- 
formance Battery (SPPB); relato de infarto agudo do miocárdio anterior ou qualquer outra doença e/ou limitação física que pudesse comprometer o desenvolvimento do protocolo dos exercícios (arritmias cardíacas avançadas, insuficiência cardíaca descompensada, entre outras); aqueles que não concluíram o período mínimo de 12 semanas de exercícios planejados no protocolo e/ou que faltaram as atividades programadas por mais de três sessões consecutivas.

Foram elegíveis para compor a amostra 138 pessoas idosas cadastradas no LANME, dos quais 60 sujeitos foram identificados como hipertensos e foram recrutados para esta pesquisa, destes, 54 preencheram os demais critérios de inclusão do estudo, assinaram o TCLE e realizaram os testes e avaliações iniciais. Entretanto, apenas 48 idosos completaram o protocolo da pesquisa (Figura 1).

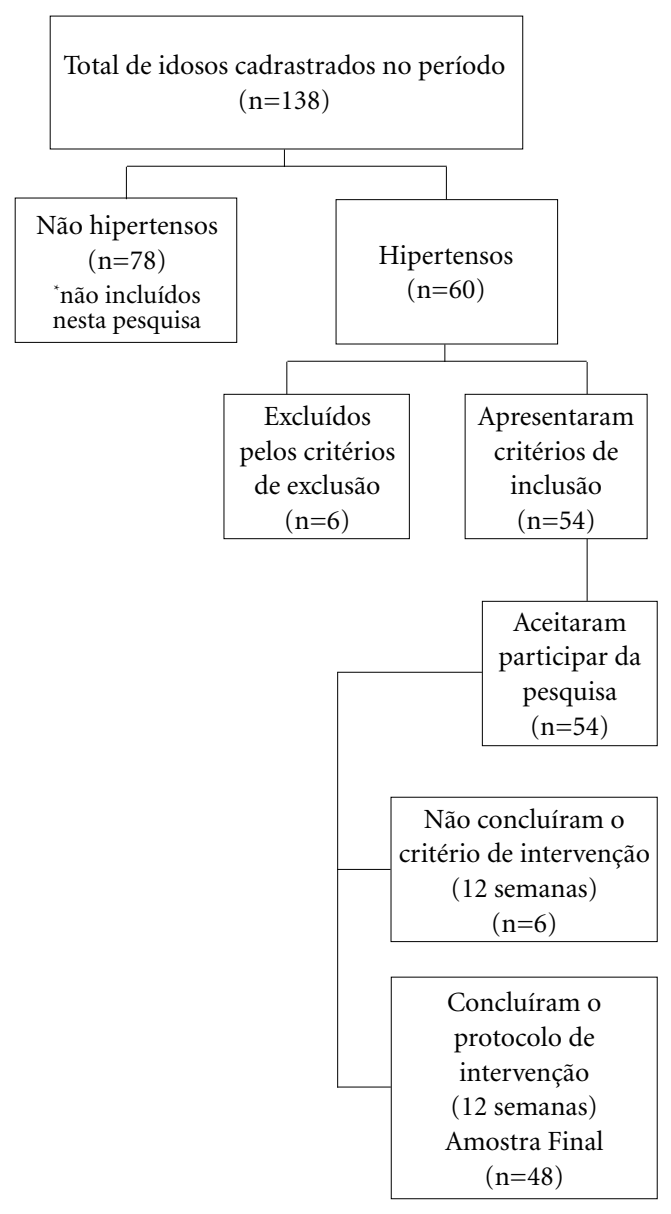

Figura 1. Fluxograma do plano de amostragem.

\section{Instrumentos e procedimentos da coleta}

O protocolo de pesquisa foi composto por variáveis sociodemográficas, características clínicas, laboratoriais e antropométricas. Avaliaramse capacidade funcional, qualidade de vida relacionada à saúde (QVRS) e presença de sintomas depressivos.

Para a medida da pressão arterial (PA) utilizou-se o aparelho digital OMRON, modelo HEM-7113 ${ }^{\circ}$ e foram adotados os padrões da I Diretriz Brasileira de Prevenção Cardiovascular ${ }^{16}$.

Para avaliação da massa corporal utilizou-se uma balança do tipo plataforma digital FILIZOLA $^{\circledR}$, calibrada, com capacidade para $200 \mathrm{~kg}$ e precisão de 100g. Os indivíduos foram pesados sem sapatos e sem adornos, mantendo-se em posição ortostática (em pé, na posição ereta, pés afastados, à largura do quadril; com o peso dividido em ambos os pés no plano de Frankfurt), ombros descontraídos e braços soltos lateralmente durante a leitura da massa corporal ${ }^{17}$.

A estatura foi estimada pela medida da altura do joelho, segundo Chumlea et al..$^{18}$, com paquímetro antropométrico com sensibilidade de $1 \mathrm{~mm}$ e capacidade máxima de $90 \mathrm{~cm}$. O indivíduo foi mantido sentado, com o joelho flexionado formando um ângulo de $90^{\circ}$.

O índice de massa corporal (IMC) foi calculado pela divisão da massa corporal (em quilogramas) pela estatura ao quadrado (em metros $)^{19}$. Foi adotado o critério de Lipschitz ${ }^{20}$ para classificação.

A circunferência da cintura (CC) foi medida com fita métrica inelástica de fibra de vidro com escala de $0,1 \mathrm{~mm}$ e capacidade máxima de $2 \mathrm{~m}$., segundo o protocolo de Martins e Lopes ${ }^{21}$ e considerados os pontos de cortes estabelecidos nas Diretrizes Brasileiras de Obesidade ${ }^{22}$.

Para análise do perfil lipídico foi coletado sangue dos participantes com o mínimo de oito horas de jejum, no laboratório de análises do Hospital Universitário Professor Alberto Antunes (HUPAA-UFAL). Utilizou-se equipamento Automação ViteK Systems Clinline 150 - Biomerieux ${ }^{\oplus}$. O colesterol total (CT), a lipoproteína de alta densidade (HDL) e os triglicerídeos (TG) foram medidos por colorimetria enzimática; a lipoproteína de baixa densidade (LDL) foi calculada pela fórmula de Friedwald et al. ${ }^{23}$. Foram adotados os valores da V Diretrizes Brasileiras sobre Dislipidemias e Prevenção da Aterosclerose ${ }^{24}$.

O risco absoluto para desenvolver eventos cardiovasculares em 10 anos foi realizado pelo Escore de Risco Global (ERG) e os indivíduos 
classificados em três grupos de risco de acordo com a I Diretriz Brasileira de Prevenção Cardiovascular ${ }^{16}$.

A QVRS foi avaliada através do questionário Short-Form-36 (SF-36) e a presença de sintomas depressivos verificada pela Escala de Depressão Geriátrica (GDS-15).

\section{Protocolo dos exercícios}

Pesquisadores, devidamente treinados, desenvolveram um protocolo misto de exercícios físicos (exercícios funcionais em circuito e caminhada) voltados para a melhora da flexibilidade, força muscular, equilíbrio, coordenação e resistência cardiorrespiratória com duração de 16 semanas e frequência de duas vezes por semana, totalizando 32 sessões. Além das duas sessões semanais, os indivíduos realizaram caminhada com duração de 60 minutos, uma vez por semana, para complementar o período mínimo estabelecido pelo American College Sports of $\mathrm{Me}$ dicine ${ }^{25}$.

O circuito foi composto por 10 estações de exercícios com objetivos específicos que variaram a cada semana (4 estações com exercícios para força muscular, 2 para flexibilidade, 2 para equilíbrio e 2 para coordenação) seguindo de forma sequencial, com início aleatório. Cada estação tinha duração de 30 segundos e os intervalos de descanso ocorriam após o término de cada rodada no circuito (3 minutos).

A intensidade dos exercícios foi avaliada através da percepção subjetiva do esforço (escala de Borg modificada por Foster et al. ${ }^{26}$ ), numa escala de 0 a 10 onde o valor máximo (10) foi considerado o maior esforço físico realizado e o valor mínimo (0) foi considerado a condição de repouso absoluto. Com a escala de Borg afixada em cada estação de exercício, os indivíduos foram orientados a permanecerem numa intensidade moderada de 5 ou 6 na escala de Borg, o que corresponde 55 a $85 \%$ da frequência cardíaca máxima $^{15,25,27}$.

Previamente às intervenções, todos participaram de uma familiarização por duas semanas, sendo orientados sobre a mecânica e a execução correta dos exercícios. A pressão arterial (PA) foi aferida antes dos exercícios e aqueles que apresentaram PA sistólica $\geq 160 \mathrm{mmHg}$ e PA diastóli$\mathrm{ca} \geq 100 \mathrm{mmHg}$ não participaram das atividades até que os níveis normalizassem.

Importa ressaltar que nenhum dos participantes recebeu acompanhamento nutricional antes ou durante a aplicação do protocolo de exercícios físicos. Posteriormente, todos foram atendidos e orientados por nutricionistas componentes do programa de extensão.

\section{Análise estatística}

Para análise estatística utilizou-se o Programa Estatístico SPSS (Statistical Package for Social Sciences) versão $20.0^{\circ}$. Foram realizados testes paramétricos e não paramétricos, considerando a distribuição das variáveis, analisado por meio do teste Shapiro-Wilk. A diferença entre as médias foi calculada pelo Teste-t pareado de Student para variáveis paramétricas e testes de Wilcoxon para variáveis não paramétricas. Os resultados foram expressos como média ou mediana \pm desvio padrão (DP) ou intervalo inter-quartil e considerados significativos quando $\mathrm{p}<0,050$. A análise de diferença de frequências antes e depois foi realizada por meio do teste de McNemar.

\section{Aspectos éticos}

O projeto seguiu os padrões exigidos pela resolução 466/2012 do Conselho Nacional de Saúde do Ministério da Saúde e aprovado pelo Comitê de Ética em Pesquisa da UFAL.

\section{Resultados}

O grupo estudado apresentou média de idade de 66,5 anos $\pm 4,8$ anos, sendo $54,2 \%$ do sexo feminino. Observou-se maior frequência de idosos com renda familiar maior ou igual a 1 salário mínimo $(70,8 \%)$ e tempo de estudo superior a 9 anos $(54,2 \%)$. Todos faziam uso de medicamentos anti-hipertensivos. Quanto à presença de fatores de risco cardiovascular, observou-se maior frequência de dislipidemia $(58,3 \%)$ e de sedentarismo $(45,8 \%)$ entre os participantes do estudo (Tabela 1).

Em relação ao perfil lipídico, houve redução dos valores médios de colesterol total $(\mathrm{p}=$ $0,0001)$ e LDL colesterol $(\mathrm{p}=0,001)$ e aumento dos valores da mediana do HDL $(\mathrm{p}=0,012)$, quando comparados os momentos pré e pós realização do protocolo de atividade física. Não foram observadas modificações nos valores médios de triglicerídeos, PAS, PAD e FC (Tabela 2).

Avaliando os dados antropométricas, não foram observadas reduções da massa corporal total $(\mathrm{p}=0,229), \operatorname{IMC}(\mathrm{p}=0,832)$ e CC $(\mathrm{p}=0,741)$ antes e após o período de realização dos exercícios (Tabela 2). 
Tabela 1. Variáveis sociodemográficas e prevalência de fatores de RCV em idosos hipertensos inseridos em um programa de extensão universitário. Maceió, 2015 $(n=24)$.

\begin{tabular}{|c|c|c|}
\hline Variáveis & $\begin{array}{c}\text { Média }( \pm \mathrm{DP}) \\
/ \mathbf{n}(\%)\end{array}$ & p-valor ${ }^{*}$ \\
\hline Idade (anos) & $66,5( \pm 4,8) /-$ & - \\
\hline Sexo & & 0,665 \\
\hline Masculino & $-/ 22(45,8)$ & \\
\hline Feminino & $-/ 26(54,2)$ & \\
\hline Estado Conjugal & & 0,665 \\
\hline $\begin{array}{l}\text { Casado ou em União } \\
\text { Consensual }\end{array}$ & $-/ 26(54,2)$ & \\
\hline $\begin{array}{l}\text { Outro (Solteiro, } \\
\text { Divorciado ou Viúvo) }\end{array}$ & $-/ 22(45,8)$ & \\
\hline Tempo de estudo & & 0,665 \\
\hline$\leq 9$ anos & $-/ 22(45,8)$ & \\
\hline$>9$ anos & $-/ 26(54,2)$ & \\
\hline Renda Familiar & & 0,006 \\
\hline Menor ou igual a 1 SM & $-/ 14(9,2)$ & \\
\hline Maior que $1 \mathrm{SM}$ & $-/ 34(70,8)$ & \\
\hline Situação Profissional & & $<0,001$ \\
\hline $\begin{array}{l}\text { Desempregado/Sem } \\
\text { Renda }\end{array}$ & $-/ 2(4,2)$ & \\
\hline $\begin{array}{l}\text { Aposentado/Com } \\
\text { Renda }\end{array}$ & $-/ 46(95,8)$ & \\
\hline Diabetes Mellitus & & $<0,001$ \\
\hline Sim & $-/ 8(16,7)$ & \\
\hline Não & $-/ 40(83,3)$ & \\
\hline Dislipidemia & & 0,312 \\
\hline Sim & $-/ 28(58,3)$ & \\
\hline Não & $-/ 20(41,7)$ & \\
\hline Tabagismo & & $<0,001$ \\
\hline Sim & $-/ 0(0,0)$ & \\
\hline Não & $-/ 48(100)$ & \\
\hline Sedentarismo & & 0,665 \\
\hline Sim & $-/ 22(45,8)$ & \\
\hline Não & $-/ 26(54,2)$ & \\
\hline Etilismo & & 0,001 \\
\hline Sim & $-/ 12(25,0)$ & \\
\hline Não & $-/ 36(75,0)$ & \\
\hline
\end{tabular}

Legenda: DP (Desvio Padrão); RCV (Risco Cardiovascular); SM (Salário Mínimo). * p-valor relativo à comparação das proporções binárias hipotetizadas $(0,5)$ para as variáveis categóricas desta amostra.

Fonte: Dados da pesquisa do presente estudo.

No início da pesquisa 70,8\% indivíduos foram classificados com alto risco para o desenvolvimento de evento cardiovascular para os próximos 10 anos versus $50,0 \%$ ao término $(\mathrm{p}=$ $0,125)$. O percentual do RCV e a pontuação do
ERG apresentaram reduções significativas após o protocolo de exercícios físicos $(\mathrm{p}=0,019$ e $\mathrm{p}=$ 0,001 , respectivamente) (Tabela 2 ).

Inicialmente $25 \%$ da amostra apresentaram sintomas depressivos, enquanto ao término essa frequência reduziu para $4,2 \%(p=0,053)$. A mediana da pontuação atribuída ao GDS reduziu de 3,63 pontos $\pm 1,75$ IQ para 1,5 pontos $\pm 1,75$ IQ $(\mathrm{p}<0,001)$.

Observaram-se melhoras significativas nos seguintes domínios do SF-36: aspectos físicos ( $\mathrm{p}$ $=0,004)$, estado geral de saúde $(p=0,032)$ e vitalidade $(\mathrm{p}=0,002)$ (Tabela 3$)$.

\section{Discussão}

A presente pesquisa foi desenvolvida com o objetivo de avaliar os efeitos da prática de exercícios físicos sobre o risco cardiovascular, qualidade de vida e presença de sintomas depressivos em idosos hipertensos, fortalecendo o papel dessa prática como adjuvante à prevenção de eventos cardiovasculares indesejáveis e apresentou-se como uma intervenção não farmacológica eficaz na redução do colesterol total e LDL-c, aumento da HDL-c e melhor qualidade de vida entre os idosos. Além dos resultados observados, a prática de exercício físico regular proporciona efeitos benéficos para o estilo de vida ativo, manutenção da capacidade funcional e da autonomia física, minimizando a degeneração provocada pelo processo de envelhecimento, com melhoria geral na saúde e qualidade de vida da população idosa ${ }^{28,29}$.

Desse modo, sendo os sujeitos deste estudo pessoas idosas hipertensas, o risco para eventos cardiovasculares é naturalmente maior que na população geral. Apesar de não haver diferença na proporção entre sexos, a maioria dos participantes desta pesquisa foram mulheres, semelhante à recente pesquisa de Pimenta e Caldeira ${ }^{6} \mathrm{com}$ predomínio do sexo feminino $(64,35 \%)$ e média da idade de 65,5 anos $\pm 10,3$ anos, corroborando com o processo de feminilização do envelhecimento. Esta representação feminina resulta da maior expectativa de vida das mulheres que, em média, vivem oito anos a mais que os homens ${ }^{30}$.

$\mathrm{Na}$ população estudada houve maior frequência de dislipidemia e grande parte relatou não praticar atividade física de lazer, fatores que somados aumentam o risco de desenvolvimento de doenças crônicas, incluindo a hipertensão. Estudos semelhantes observam que o sedentarismo e a dislipidemia coexistem em alta frequência na população idosa ${ }^{1,31}$. 
Tabela 2. Características clínicas, laboratoriais, antropométricas e número de fatores de risco cardiovascular em idosos hipertensos do Programa de Extensão Exercitando a Melhor Idade da UFAL. Maceió, 2015 (n=24).

\begin{tabular}{|c|c|c|c|c|}
\hline Variáveis & $\begin{array}{l}\text { Pré-Intervenção } \\
\text { Média ( } \pm \mathrm{DP}) / \\
\text { Mediana ( } \pm \mathrm{IQ})\end{array}$ & $\begin{array}{c}\text { Pós-Intervenção } \\
\text { Média ( } \pm \text { DP) / } \\
\text { Mediana ( } \pm \text { IQ) }\end{array}$ & $\mathrm{IC}_{95 \%}$ & p-valor \\
\hline $\mathrm{CT}(\mathrm{mg} / \mathrm{dl})^{\mathrm{a}}$ & $203,88(46,1)$ & $188,08(43,6)$ & $8,11-21,88$ & $<0,001^{\dagger}$ \\
\hline $\mathrm{HDL}(\mathrm{mg} / \mathrm{dl})^{\mathrm{b}}$ & $44,50(14,5)$ & $48,00(28,7)$ & - & $0,012^{\#}$ \\
\hline $\mathrm{LDL}(\mathrm{mg} / \mathrm{dl})^{\mathrm{a}}$ & $133,49(45,2)$ & $115,95(41,8)$ & $4,87-30,20$ & $0,009^{\dagger}$ \\
\hline TGL (mg/dl) ${ }^{\mathrm{b}}$ & $161,00(93,0)$ & $160,75(66,5)$ & - & $0,330^{\#}$ \\
\hline PAS $(\mathrm{mmHg})^{\mathrm{b}}$ & $141,00(25,5)$ & $132,50(21,3)$ & - & $0,107^{\#}$ \\
\hline $\operatorname{PAD}(\mathrm{mmHg})^{\mathrm{b}}$ & $83,50(19,5)$ & $79,00(14,5)$ & - & $0,210^{\#}$ \\
\hline $\mathrm{FC}(\mathrm{bpm})^{\mathrm{b}}$ & $70,00(5,0)$ & $72,00(11,0)$ & - & $0,586^{\#}$ \\
\hline Peso $(\mathrm{kg})^{\mathrm{a}}$ & $75,03(15,7)$ & $74,33(14,7)$ & $-0,47-1,87$ & $0,229^{\dagger}$ \\
\hline $\operatorname{IMC}\left(\mathrm{kg} / \mathrm{m}^{2}\right)^{\mathrm{a}}$ & $28,87(4,9)$ & $28,79(4,18)$ & $-0,65-0,80$ & $0,832^{\dagger}$ \\
\hline $\mathrm{CC}(\mathrm{cm})^{\mathrm{a}}$ & $92,31(11,6)$ & $91,97(10,5)$ & $-1,75-2,43$ & $0,741^{\dagger}$ \\
\hline Pontos do ERG ${ }^{\text {a }}$ & $15,62(3,1)$ & $14,79(3,02)$ & $1,27-3,89$ & $0,001^{\dagger}$ \\
\hline Percentual de RCV ${ }^{a}$ & $19,47(7,5)$ & $17,01(7,3)$ & $0,45-4,47$ & $0,019^{\dagger}$ \\
\hline
\end{tabular}

Legenda: ${ }^{\mathrm{M} M e ́ d i a ~(~} \pm$ Desvio Padrão - DP); ${ }^{\mathrm{b}}$ Mediana ( \pm Intervalo Interquartil - IQ). IC ${ }_{950}$ : Intervalo de Confiança. ${ }^{\circ}$ Teste $t$-pareado. \#Teste de Wilcoxon. CT - Colesterol Total; HDL - Hight Density Lipoprotein; LDL - Low Density Lipoprotein; TGL - Triglicerídeos; PAS - Pressão Arterial Sistólica; PAD - Pressão Arterial Diastólica; FC - Frequência Cardíaca; IMC - Índice de Massa Corporal; CC - Circunferência da Cintura; FRC - Fatores de Risco Cardiovascular; ERG - Escore de Risco de Global; RCV - Risco Cardiovascular. Fonte: Dados da pesquisa do presente estudo.

Tabela 3. Valores dos domínios da QVRS avaliados pelo SF-36 em idosos hipertensos inseridos em um programa de extensão universitário. Maceió, 2015 ( $\mathrm{n}=24)$.

\begin{tabular}{lcccc}
\hline \multicolumn{1}{c}{ Domínios } & $\begin{array}{c}\text { Pré-Intervenção } \\
\text { Média }( \pm \text { DP) }\end{array}$ & $\begin{array}{c}\text { Pós-Intervenção } \\
\text { Média }( \pm \text { DP) }\end{array}$ & IC $_{95 \%}$ & p-valor $^{*}$ \\
\hline CF & $63,95( \pm 26,7)$ & $73,54( \pm 21,1)$ & $-19,94-0,77$ & 0,068 \\
AF & $61,19( \pm 29,4)$ & $76,56( \pm 20,7)$ & $-25,20--5,52$ & 0,004 \\
DOR & $65,27( \pm 26,3)$ & $69,16( \pm 21,2)$ & $-15,34-7,56$ & 0,490 \\
EGS & $65,77( \pm 16,4)$ & $73,50( \pm 19,9)$ & $-14,74--0,70$ & 0,032 \\
VT & $68,22( \pm 21,4)$ & $80,46( \pm 13,2)$ & $-19,47--5,00$ & 0,002 \\
AS & $82,50( \pm 23)$ & $89,94( \pm 16,7)$ & $-19,46-4,57$ & 0,213 \\
AE & $76,73( \pm 27,9)$ & $78,47( \pm 23,04)$ & $-12,56-9,09$ & 0,743 \\
SM & $86,04( \pm 18,05)$ & $89,58( \pm 14,3)$ & $-11,30-4,22$ & 0,355 \\
\hline
\end{tabular}

Legenda: $\mathrm{IC}_{950}$ : Intervalo de Confiança. ${ }^{*}$ Test t-pareado. CF - Capacidade Funcional; AF - Aspectos Físicos; DOR - Dor Corporal; ESG - Estado Geral da Saúde; VT - Vitalidade; AS - Aspectos Sociais; AE - Aspectos Emocionais; SM - Saúde Mental. Fonte: Dados da pesquisa do presente estudo.

O risco cardiovascular estratificado pelo ERG, no início desta pesquisa, indicou que mais de dois terços dos idosos foram classificados com alto risco para o desenvolvimento de evento cardiovascular nos próximos 10 anos. Ao término da aplicação do protocolo misto de exercícios físicos, muitos daqueles que se encontravam com alto risco, classificaram-se como risco moderado. Infere-se que este resultado pode estar pautado na redução de fatores de risco e melhora do perfil lipídico.
Semelhante ao presente estudo, pesquisadores identificaram reduções significativas no RCV de mulheres obesas na pós-menopausa e sedentárias com média de idade de 61,3 anos $\pm 6,4$ anos, em resposta a um protocolo de exercícios físicos ${ }^{29}$.

Campos et al. ${ }^{32}$ investigaram o efeito do exercício físico aeróbio e resistido de intensidade moderada em idosos de ambos os sexos durante 17 semanas. Foram observadas diferenças significativas nos TG $(-11 \%)$, CT $(-6 \%)$, LDL $(-13 \%)$, 
HDL (+5\%), concluindo que os exercícios resultaram em mudanças positivas desses importantes fatores de risco cardiovasculares.

Estudo realizado com o objetivo de avaliar o impacto do exercício físico sobre o perfil lipídico em pessoas idosas após 8 semanas observou melhora significativa nos níveis de CT, LDL, HDL e TG ao final do programa ${ }^{33}$. Marques et al. ${ }^{34}$ avaliaram os efeitos do exercício multicomponente (exercício aeróbio, exercícios resistidos e exercícios voltados para melhorar o equilíbrio e flexibilidade) durante 48 semanas de treinamento, constatando que o exercício multicomponente foi eficaz para a melhora do perfil lipídico com a redução significativa do TG, LDL e aumento nos níveis de HDL. Importa observar que nesses estudos o período de aplicação dos protocolos de exercícios variou de 8 a 48 semanas. Entretanto, mesmo em períodos mais curtos, resultados benéficos à saúde foram observados.

Pesquisas relatam os efeitos benéficos do exercício regular, produzindo modificações no metabolismo lipídico e lipoproteico, observando-se aumento nos níveis de $\mathrm{HDL}^{35}$ e uma diminuição na composição do colesterol total e LDL circulante, associado à atividade enzimática ${ }^{36}$. Tais modificações parecem estar associadas ao aumento da cinética de LDL-c na circulação, o que prolonga o período em que o LDL-c permanece na forma reduzida $^{37}$. Portanto, indivíduos fisicamente ativos apresentam níveis séricos mais baixos de CT, TG e LDL e concentrações mais elevadas de HDL em relação aos inativos. As alterações séricas de HDL são atribuídas à diminuição de sua degradação no fígado e aumento de sua síntese ${ }^{36}$.

Segundo metanálise ${ }^{38}$, o treinamento de forca pode reduzir a pressão sistólica e diastólica em aproximadamente $3 \mathrm{mmHG}$ e $4 \mathrm{mmHg}$, respectivamente. Estudo realizado por Molmen-Hansen et al. $^{39}$ demonstrou que uma combinação intermitente de alta intensidade (> 80\% para $60 \%$ de frequência cardíaca máxima) de treinamento aeróbio foi mais eficaz do que o treinamento contínuo com intensidade moderada no estágio 1 da hipertensão.

O American College de Medicina Esportiva, a Sociedade Europeia de Hipertensão e a Sociedade Europeia de Cardiologia recomendam que o tipo primário de exercício para o manejo da pressão sanguínea deve ser aeróbico, complementado por treinamento de resistência ${ }^{40,41}$. Nesta pesquisa, nenhuma modificação foi observada nas médias da pressão arterial.

O impacto da prática de atividades físicas na vida das pessoas merece considerações e reflexões profundas no contexto da Educação Física, uma vez que proveem ganhos na saúde, tais como: redução do risco de sarcopenia, incremento da força muscular e da densidade óssea, melhora do equilíbrio, diminuição da pressão arterial, desenvolvimento dos aspectos psicológicos e sociais, melhora da autoestima, diminuição do estresse, melhora das funções cognitivas e da socialização, além de prevenir quedas ${ }^{42,43}$. Pode também ser uma atividade de lazer, especialmente para idosos que terão a oportunidade de expressar sentimentos, pensamentos, concepções, de interagir com a sociedade e reforçar seus laços afetivos e culturais $^{44}$.

Brunoni et al. ${ }^{45}$ verificaram os efeitos do treinamento de força durante 12 semanas e frequência de duas vezes por semana sobre a QVRS e sintomas depressivos em 24 idosas com média de idade de 65,6 anos \pm 4,5 anos e observaram diferenças significativas nos escores do SF-36 após o período de treinamento nos domínios CF, EGS, VIT e SM. Já em relação aos sintomas depressivos, foi encontrada uma redução na pontuação do GDS do período pré-treinamento para o póstreinamento (9,5 pontos $\pm 4,2$ pontos versus 6 pontos $\pm 6,7$ pontos; $\mathrm{p}<0,001)$ e uma redução na frequência de indivíduos com elevado risco para depressão de $45,8 \%$ para $16,7 \%$.

No presente estudo, houve melhora nos domínios AF, EGS e VIT e importante redução na média da pontuação do GDS, além de redução significativa na frequência de indivíduos com sintomas depressivos ( $\geq$ a 5 pontos). Melo et al. ${ }^{12}$ investigaram o efeito do exercício físico (aeróbio e força) nos domínios de QVRS do SF-36 em idosos com depressão leve ou moderada e após três meses de intervenção foi observada uma melhora dos domínios AF, DOR, AS e SM, além da redução significativa dos sintomas depressivos.

Os resultados acima citados foram corroborados na investigação de Denk et al. ${ }^{46}$ ao concluírem que indivíduos praticantes de atividade física apresentam autopercepção de melhor estado de saúde. Importa salientar que saúde percebida não é simplesmente ausência de doença, mas está fortemente influenciada pelo grau de incapacidade funcional e determinados fatores relacionados à personalidade ${ }^{47}$. Bem-Ezra \& Shmotkin ${ }^{48}$ inferem que a autopercepção de saúde declina com o avanço da idade e que se associa à maior mortalidade, pior saúde mental, pior funcionalidade e maior utilização dos serviços de saúde.

Na população estudada não houve reduções significativas nas médias de PA, FC, TG, massa corporal, IMC e CC. Embora a perda de peso 
resulte em redução da pressão arterial, alguns estudos demonstraram que a resposta da pressão arterial ao treinamento não depende de mudanças na massa corporal ${ }^{41}$.

Este estudo apresentou limitações, como a ausência de um grupo controle ou com protocolo de atividade física diferenciada, o que poderia levar a conclusões mais consistentes, além de avaliar a associação entre as variáveis e buscar compreender as relações de causalidade e efeito entre as mesmas. A amostra utilizada foi reduzida devido aos critérios de inclusão e exclusão desta pesquisa, fato que limita os achados.

Tais achados fortalecem a ideia da prática da atividade como promotora da saúde e qualidade de vida para o idoso nos diferentes aspectos, sejam eles físicos, psicológicos ou sociais. Consideramos importante destacar que o Programa Academia da Saúde, lançado em 2011 com o objetivo de promover práticas de exercícios e atividade fí- sica, além de contribuir para modos de vida saudáveis pode assumir importantes efeitos sobre a longevidade, devendo ser mais incentivado para sua execução efetiva e qualificada nos municípios brasileiros.

\section{Conclusão}

O protocolo misto de exercícios físicos de intensidade moderada, realizado durante 12 semanas, constituiu-se em uma alternativa viável e eficaz na redução do risco para o desenvolvimento de eventos cardiovasculares nos próximos dez anos, além de favorecer a QVRS e a redução de sintomas depressivos nesta população. Recomenda-se, portanto, um programa prolongado de exercícios físicos, associado ao acompanhamento nutricional.

\section{Colaboradores}

NA Cassiano, TS Silva e CQ Nascimento contribuíram com a redação do artigo. EM Wanderley contribuiu com a revisão crítica do artigo. ES Prado, CS Mello e TMM Santos contribuíram com a redação final e aprovação da versão a ser publicada. JA Barros-Neto trabalhou na concepção, delineamento, análise e interpretação dos dados. 


\section{Referências}

1. Campos MO, Rodrigues-Neto JF, Silveira MF, Neves DMR, Vilhena JM, Oliveira JF, Magalhães JC, Drumond D. Impacto dos fatores de risco para doenças crônicas não transmissíveis na qualidade de vida. Cien Saude Colet 2013; 18(3):873-882.

2. Silva MS, Silva NB, Alves AGP, Araújo SP, Oliveira AC. Risco de doenças crônicas não transmissíveis na população atendida em programa de educação nutricional em Goiânia (GO), Brasil. Cien Saude Colet 2014; 19(5):1409-1418.

3. Malta DC, Iser BPM, Claro RM, Moura L, Berna RTI, Nascimento AF, Silva Jr JB, Monteiro CA. Prevalência de fatores de risco e proteção para doenças crônicas não transmissíveis em adultos: estudo transversal, Brasil, 2011. Epidemiol Serv Saude 2013; 22(3):423434.

4. Matsudo SMMM. Envelhecimento, atividade física e saúde. Bol Inst Saude 2009; 47:76-79.

5. Stella F, Gobbi S, Corazza DI, Costa JLR. Depressão no idoso: diagnóstico, tratamento e benefícios da atividade física. Motriz 2002; 8(3):91-98.

6. Pimenta HB, Caldeira AP. Fatores de risco cardiovascular do escore de Framingham entre hipertensos assistidos por equipes de saúde da família. Cien Saude Colet 2014;19(6):1731-1739.

7. Soares TS. Hábitos alimentares, atividade física e escore de risco global de Framingham na síndrome metabólica. Arq Bras Cardiol 2014; 102(4):374-382.

8. Malta DC, Moura L, Prado RR, Escalante JC, Schmidt MI, Duncan BB. Mortalidade por doenças crônicas não transmissíveis no Brasil e suas regiões, 2000 a 2011. Epidemiol Serv Saude 2014; 23(4):599-608.

9. Zaar A, Reis VM, Sbardelotto ML. Efeitos de um programa de exercícios físicos sobre a pressão arterial e medidas antropométricas. Rev Bras Med Esporte 2014; 20(1):13-16.

10. World Health Organization (WHO). Global recommendations on physical activity for health. Genebra: WHO; 2010

11. Sociedade Brasileira de Cardiologia (SBC). VII Diretrizes Brasileiras de Hipertensão. Arq Bras Cardiol 2016; 107(3 Supl. 3):1-103.

12. Melo B, Moraes HS, Silveira H, Olivera N, Deslandes AC, Laks J. Efeito do treinamento físico na qualidade de vida em idosos com depressão maior. Rev Bras Ativ Fis Saude 2014; 19(2):205-214.

13. Fagard RH. Physical activity, physical fitness and the incidence of hypertension. J Hypertens 2005; 23(2):265-267.

14. Bündchen DC, Schenkel IC, Santos Z, Carvalho T. Exercício físico controla pressão arterial e melhora qualidade de vida. Rev Bras Med Esporte 2013; 19(2):91-95.

15. Nóbrega ACL, Freitas EV, Oliveira MAB, Leitão MB, Lazzoli JK, Nahas RM, Baptista CAS, Drummond FA, Rezende L, Pereira J, Pinto M, Radominski RB, Leite N, Thiele ES, Hernandez AJ, Araújo CGS, Teixeira JAC, Carvalho T, Borges SF, Rode EH. Posicionamento Oficial da Sociedade Brasileira de Medicina do Esporte e da Sociedade Brasileira de Geriatria e Gerontologia: Atividade Física e Saúde no Idoso. Rev Bras Med Esporte 1999;5(6):207-211.
16. Sociedade Brasileira de Cardiologia (SBC). I Diretriz Brasileira de Prevenção Cardiovascular. Arq Bras Cardiol 2013;101(6 Supl. 2):1-78.

17. Lohman TG. Advances in body composition assessment. Cad Saude Publica 1993; 9(Supl. 1):114-117.

18. Chumlea WC, Roche AF, Steinbaugh ML. Estimating stature from knee height for persons 60 to 90 years of age. J Am Geriatr Soc 1985; 33(2):116-120.

19. World Health Organization (WHO). Global atlas on cardiovascular disease prevention and control: policies, strategies, and interventions. Genebra: WHO; 2011.

20. Lipschitz DA. Screening for nutritional status in the elderly. Prim Care 1994; 21(1):55-67.

21. Martins MO, Lopes MA. Antropometria, técnicas e padronização. Porto Alegre: Editora Pallotti; 2003.

22. Associação Brasileira para o Estudo da Obesidade e da Síndrome Metabólica (ABESO). Diretrizes Brasileiras de Obesidade. $4^{\circ}$ ed. São Paulo: ABESO; 2016.

23. Friedwald WT, Levy RI, Fredricksin DS. Estimation of the concentration of low-density lipoprotein cholesterol in plasma, without use of the preparative ultracentrifuge. Clin Chem 1972; 18(6):499-502.

24. Sociedade Brasileira de Cardiologia (SBC). Atualização da Diretriz Brasileira de Dislipidemia e Prevenção da Aterosclerose. Arq Bras Cardiol 2017; 109(2 Supl. 1):1-91.

25. Nelson ME, Rejeski WJ, Blair SN, Duncan PW, Judge JO, King AC, Macera CA, Castaneda-Sceppa C. Physical activity and public health in older adults: recommendation from the American College of Sports Medicine and the American Heart Association. Med Sci Sports Exerc 2007; 39(8):1435-1445.

26. Foster C, Florhaug J, Franklin J, Gottschall L; Hirovatin L, Parker S, Doleshal P, Dodge C. A new approach to monitoring exercise training. J Strength Cond Res 2001; 15(1):109-115.

27. Nakamura FY, Moreira A, Aoki MS. Monitoramento da carga de treinamento: a percepção subjetiva do esforço da sessão é um método confiável? Reveducfis 2010; 21(1):1-11.

28. Freitas CMSM. Aspectos motivacionais que influenciam a adesão e manutenção de idosos a programas de exercícios físicos. Rev Bras Cineantropom Desempenho Hum 2007; 9(1):92-100.

29. Chagas EFB, Bonfim MF, Brondino NCM, Monteiro HL. Exercício físico e fatores de risco cardiovasculares em mulheres obesas na pós-menopausa. Rev Bras Med Esporte 2015;21(1):65-69.

30. Bandeira L, Melo HP, Pinheiro LS. Mulheres em dados: o que informa a PNAD/IBGE. In: Brasil. Secretaria de Políticas para as Mulheres. Edição Especial da Revista do Observatório Brasil da Igualdade de Gênero. Brasília: Secretaria de Políticas para as Mulheres; 2009. p. 107-119.

31. Zaitune MPA, Barros MBA, César CLG, Carandina L, Goldbaum M. Hipertensão arterial em idosos: prevalência, fatores associados e práticas de controle no Município de Campinas, São Paulo, Brasil. Cad Saude Publica 2006; 22(2):285-294. 
32. Campos ALP, Del Ponte LS, Cavalli AD, Afonso MRA, Schild JFG, Reichert FF. Efeito sobre o treinamento concorrente sobre o aspecto da saúde de idosas. Rev Bras Cineantropom Desempenho Hum 2013; 15(4):437-447.

33. Boardley D, Fahlman M, Topp R, Morgan AL, McNevin $\mathrm{N}$. The impact of exercise training on blood lipids in older adults. Am J Geriatr Cardiol 2007; 16(1):3035.

34. Marques E, Carvalho J, Soares JM, Marques F, Et Mota J. Efeitos da resistência e exercício físico multicomponente em perfil lipídico de mulheres mais velhas. Maturitas 2009; 63(1):84-88.

35. Iborra RT, Ribeiro IC, Neves MQ, Charf AM, Lottenberg SA, Negrão CE, Nakandakare ER, Passarelli M. Aerobic exercise training improves the role of high-density lipoprotein antioxidant and reduces plasma lipid peroxidation in type 2 diabetes mellitus. Scand J Med Sci Sports 2008; 18(6):742-750.

36. Zanella AM, Souza DRS, Godoy MF. Influência do exercício físico no perfil lipídico e estresse oxidativo. Arq Cien Saude 2007; 14(2):107-112.

37. Ribeiro IC, Iborra RT, Neves MQ, Lottenberg SA, Charf AM, Nunes VS, Negrão CE, Nakandakare ER, Quintão EC, Passarelli M. HDL atheroprotection by aerobic exercise training in type 2 diabetes mellitus. Med Sci Sports Exerc 2008; 40(5):779-786.

38. Ketelhut RG, Franz IW, Scholze J. Regular exercise as an effective approach in antihypertensive therapy. Med Sci Sports Exerc 2004; 36:4-8.

39. Molmen-Hansen HE, Stolen T, Tjonna AE, Aamot IL, Ekeberg IS, Tyldum GA, Wisloff U, Ingul CB, Stoylen A. Aerobic interval training reduces blood pressure and improves myocardial function in hypertensive patients. Eur J Prev Cardiol 2012; 19:151-160.

40. Whelton SP, Chin A, Xin X, He J. Effect of aerobic exercise on blood pressure: a meta-analysis of randomized, controlled trials. Ann Intern Med 2002; 136:493-503.

41. Hudnut F. Comment on "beyond medications and diet: alternative approaches to lowering blood pressure: a scientific statement from the American Heart Association". Hypertension 2014; 63:3.
42. Taeffe DR. Sarcopenia: exercise as a treatment strategy. Aust Fam Physician 2006; 35(3):130-134.

43. Samulski DM, Noce F. A importância da atividade física para a saúde e qualidade de vida: um estudo entre professores, alunos e funcionários da UFMG. Rev Bras Ativ Fis Saude 2000; 5(1):5-21.

44. Bramante AC. Lazer: concepções e significados. Licere 1998; 1(1):9-17.

45. Brunoni L, Schuch FB, Bias CP, Kruel LFM, Tiggemann CL. Treinamento de forca diminui os sintomas depressivos e melhora a qualidade de vida relacionada a saúde em idosas. Rev Bras Educ Fís Esporte 2015; 29(2):189-196.

46. Denk H, Pache D, Schaller HJ. Handbuch Altessport. Schordnorf: Hofmann Verlag: 2003.

47. Sposito G, D’Elboux Diogo MJ, Cintra AP, Neri AL, Guariento ME, De Sousa MLR. Relações entre o bem-estar subjetivo e a funcionalidade em idosos em seguimento ambulatorial. Rev Bras Fisioter 2010; 14(1):81-89.

48. Bem-Ezra M, Shmotkin D. Predictors of mortality in the old-old in Israel: the Cross-sectional and Longitudinal Aging Study. J Am Geriatr Soc 2006; 54(6):906911.

Artigo apresentado em 18/07/2018

Aprovado em 14/11/2018

Versão final apresentada em 16/11/2018 